\title{
Constituintes químicos das folhas e do caule de Coccoloba mollis Casaretto (Polygonaceae)
}

\author{
Patrícia Emanuella S. Oliveira, ${ }^{1}$ Wagner S. dos Santos, ${ }^{1}$ Lucia M. Conserva, ${ }^{* 1}$ \\ Rosangela P. de Lyra Lemos ${ }^{2}$
}

\author{
${ }^{1}$ Instituto de Química e Biotecnologia, Universidade Federal de Alagoas, 57072-970 Maceió-AL, Brasil, \\ ${ }^{2}$ Instituto do Meio Ambiente do Estado de Alagoas, 57017-320 Maceió-AL, Brasil
}

\begin{abstract}
RESUMO: O estudo químico das frações neutra em hexano das folhas e em diclorometano do caule de Coccoloba mollis resultou no isolamento de um triterpeno pentacíclico (simiarenol), que pela primeira vez ocorre em Polygonaceae, dois fitoesteróides (sitostenona e sitosterol), um diterpeno (trans-fitol) e de um benzenóide (ácido vanílico) que está sendo descrito pela vez neste gênero. A identificação estrutural destes compostos foi feita com base na análise dos dados espectrais (IV, EM e RMN, incluindo DEPT e HMQC), bem como pela comparação com dados descritos na literatura.
\end{abstract}

Unitermos: Coccoloba mollis, Polygonaceae, triterpeno, benzenóide, fitoesteróides, diterpeno.

\begin{abstract}
Chemical constituents from leaves and stem of Coccoloba mollis Casaretto (Polygonaceae)". This work describes the isolation of a pentacyclic triterpene (simiarenol), described for the first time in this family, two phytosteroids (sitostenone and sitosterol), a diterpene (trans-phytol) and a benzenoid (vanillic acid), described for the first time in this genus, from leaves and stem of Coccoloba mollis. These compounds were identified on basis of their spectral data (IR, MS and NMR, including DEPT and HMQC), as well as by comparison with literature data.
\end{abstract}

Keywords: Coccoloba mollis, Polygonaceae, triterpene, benzenoid, phytosteroids, diterpene.

\section{INTRODUÇÃO}

A família Polygonaceae, única representante da ordem Polygonales (Barroso, 1978), compreende cerca de 40 gêneros, com mais de 800 espécies distribuídas nas regiões tropicais, temperadas e subtropicais. No Brasil, sete desses gêneros têm ocorrência espontânea, sendo Coccoloba o mais representativo (Melo, 2000). Algumas espécies desta família são cultivadas para ornamentação ou como fontes forrageiras (Melo, 1999) e muitas são utilizadas como medicinais (Jácome et al., 2004; Falcão et al., 2005; Barbosa-Filho et al., 2006a,b; Cardoso et al., 2006; Agra et al., 2007; Agra et al., 2008; Barbosa-Filho et al., 2008).

O gênero Coccoloba consiste de cerca de 400 espécies de distribuição neotropical, das quais 45 ocorrem no Brasil. Possui grande interesse florístico, uma vez que suas espécies ocorrem em diferentes formações vegetais, sendo algumas indicadas como possíveis marcadores fitogeográficos (Melo, 2004). Muitas dessas espécies são úteis do ponto de vista etnomedicinal. Dentre as quais estão as raízes de C. uvifera L. em anemia após o parto (Morton, 1977) e as cascas e as folhas em úlceras, erupção da pele e como digestivas (Coe \& Anderson, 1996), as folhas de C. barbadensis Jacq. para problemas renais (Zamora-Martinez \& Pola, 1992) e as cascas de $C$. diversifolia Jacq. como analgésica e os frutos como antidiarréico (Halberstein \& Saunders, 1978). Por sua vez, os ensaios biológicos até então efetuados revelaram ação antifúngica em C. acrosticoides (Cota et al., 2003) e C. dugandiana A.C. Smith (Li et al., 1999), antimalárica em C. pubescens L. (Antoun et al., 1993), antibacteriana em C. acrosticoides Cham. (Cota et al., 2003), C. cozumelensis Hemsl. (Perez et al., 2001) e C. uvifera (Frisbey et al., 1953), antimutagênica em C. densifrons Mart. (Wall et al., 1988), inibitória da topoisomerase II em C. hondurensis Lundell. (Wall et al., 1996), moluscicida em C. cordifolia Meisn. (Sousa \& Rouquayrol, 1974) e aleloquímica em C. uvifera (Keiser et al., 1975). Em relação ao perfil químico do gênero Coccoloba, as espécies até então investigadas estão representadas por flavonóides (Compagnone et al., 1995; Xing-Cong et al., 1999), terpenóides (Dan \& Dan, 1986; Cota et al., 2003), benzenóide (Xing-Cong et al., 1999) e ácidos carboxílicos e ésteres (Shaw et al., 1992).

A espécie Coccoloba mollis (sin. C. polystachya Weddel), conhecida como "folha-de-bôlo" e "falsonovateiro” (Lorenzi, 1998), ocorre nas Américas Central e do Sul. No Brasil, distribui-se em todas as regiões 


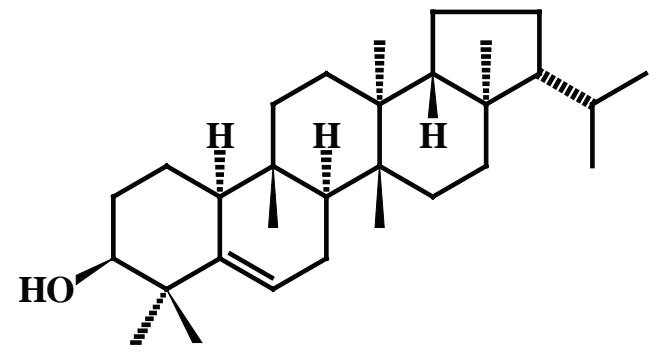

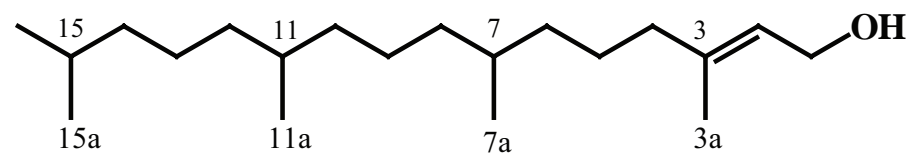

1

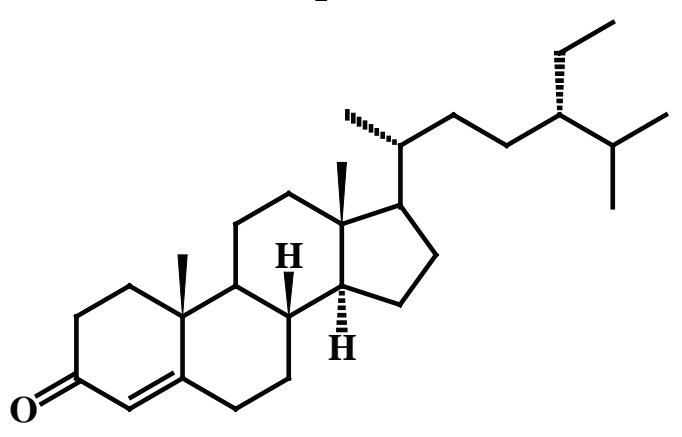

3<smiles>CC[C@H](CC[C@@H](C)C1CC[C@H]2C3CC=C4CC(O)CCC4(C)C3CCC12C)C(C)C</smiles>

4<smiles>COc1cc(C(=O)O)ccc1O</smiles>

Figura 1. Constituintes químicos de Coccoloba mollis.

(Melo, 2004), sendo recomendada para a composição de reflorestamentos mistos destinados à recuperação vegetal em áreas degradadas (Lorenzi, 1998). No estado do Paraná, Brasil, esta espécie é usada como modulador de memória e energético (Barros et al., 2007) e, com o nome de "Erva da memória", em formulações fitoterápicas para estresse, insônia, anemias, queda de visão e impotência sexual (Ferreira et al., 2006). Os estudos químicos referentes a esta espécie relatam à ocorrência nas folhas de hidrocarbonetos $\left(\mathrm{C}_{16}\right.$ a $\mathrm{C}_{31}$, exceto $\mathrm{C}_{17}$ ) e de um triterpeno (3-taraxenona) (Ferreira et al., 2006) e de antraquinonas (fissiona e emodina) nas raízes (Barros et al., 2007). O presente trabalho relata a primeira ocorrência de um triterpeno pentacíclico na família Polygonaceae (1), um diterpeno (2), dois fitoesteróides (3-4) e de um benzenóide (5) nas folhas e caule desta espécie (Figura 1).

\section{MATERIAL E MÉTODOS}

\section{Métodos gerais}

As cromatografias em coluna foram efetuadas em gel de sílica 60 G (70-230 e 230-400 mesh, Merck), 60 H (Merck) e em Sephadex LH-20 (Pharmacia), enquanto que nas cromatografias em camada delgada analítica e preparativa $(0,25$ e $0,75 \mathrm{~mm}$ de espessura, respectivamente) utilizou-se gel de sílica $60 \mathrm{PF}_{254}$ (Merck). Os espectros IV foram registrados em pastilhas de $\mathrm{KBr}$ utilizando espectrofotômetro Perkin-Elmer, FTIR 1750, contendo $1 \%$ da amostra. Os espectros de RMN $\left({ }^{1} \mathrm{H}: 200\right.$ e $500 \mathrm{MHz} ;{ }^{13} \mathrm{C}$ : 50 e $125 \mathrm{MHz}$ ) foram obtidos em espectrômetros Varian Mercury-200 e DRX-500, respectivamente. O sinal residual do solvente ou o TMS foram utilizados como referência interna. $\mathrm{O}$ espectro de massas foi registrado em um cromatográfo líquido acoplado a um espectrômetro de massas (LC-EM) da Shimadzu operando no modo positivo por ionização química à pressão atmosférica (APCI).

\section{Material vegetal}

As folhas e o caule de Coccoloba mollis Casar foram coletados em março de 2003, no município de São Miguel dos Campos, Alagoas, Brasil. A identificação botânica da espécie foi efetuada por Rosângela Pereira de Lyra Lemos do Instituto do Meio Ambiente do Estado de Alagoas, onde a exsicata do material herborizado foi depositada (MAC-17.274). 


\section{Extração e isolamento dos constituintes químicos}

As folhas (643 g) e o caule (5750 g), após secagem à temperatura ambiente e trituração, foram individualmente macerados com acetona e/ou etanol $90 \%$. Os extratos brutos obtidos, após concentração das soluções em evaporador rotatório [folhas: acetona (8,33 g), EtOH (7,38 g); caule: EtOH (114,0 g)], foram suspensos em solução $\mathrm{MeOH}-\mathrm{H}_{2} \mathrm{O}$ (3:2) e extraídos sucessivamente com $\mathrm{C}_{6} \mathrm{H}_{14}, \mathrm{CH}_{2} \mathrm{Cl}_{2}$ e AcOEt. O extrato em $\mathrm{C}_{6} \mathrm{H}_{14}$ (5,35 g), oriundo da partição do extrato em acetona das folhas, foi tratado sob agitação durante 2 horas com $\mathrm{NaOH}$ a $2 \%$ e extraído com $\mathrm{C}_{6} \mathrm{H}_{14}$. Após eliminação do solvente em evaporador rotatório obtevese a fração neutra em $\mathrm{C}_{6} \mathrm{H}_{14}(3,98 \mathrm{~g})$. Esta fração, após sucessivos fracionamentos cromatográficos em gel de sílica (230-400 mesh e $60 \mathrm{H}$ ), utilizando misturas de $\mathrm{C}_{6} \mathrm{H}_{14}$-AcOEt em gradiente crescente de polaridade como eluentes, sucessivas recristalizações com $\mathrm{MeOH}$ e CCD preparativa [sílica gel, $\mathrm{C}_{6} \mathrm{H}_{14}$-AcOEt (97:3) contendo gotas de $\mathrm{MeOH}$ ] conduziu ao isolamento de simiarenol (1, $115 \mathrm{mg})$, trans-fitol (2, $56 \mathrm{mg})$, sitostenona (3, 63 $\mathrm{mg}$ ) e sitosterol (4, $64 \mathrm{mg})$.

$\mathrm{O}$ extrato em $\mathrm{CH}_{2} \mathrm{Cl}_{2}(7,28 \mathrm{~g})$, proveniente da partição do extrato em EtOH do caule, foi filtrado em gel de sílica (70-230 mesh), utilizando solventes de diferentes polaridades: $\mathrm{C}_{6} \mathrm{H}_{14}(0,12 \mathrm{~g}), \mathrm{C}_{6} \mathrm{H}_{14}-\mathrm{CH}_{2} \mathrm{Cl}_{2}$ 1:1 (0,33 g), $\mathrm{CH}_{2} \mathrm{Cl}_{2}$ (0,84 g), $\mathrm{CH}_{2} \mathrm{Cl}_{2}$-AcOEt 1:1 (1,96 g), AcOEt (0,46 g) e MeOH (2,42 g). A subfração em $\mathrm{CH}_{2} \mathrm{Cl}_{2}(0,84 \mathrm{~g})$ após permeação em gel (Sephadex LH$20 \mathrm{com} \mathrm{MeOH}$ ) e fracionamento em gel de sílica $60 \mathrm{H}$ com misturas de $\mathrm{C}_{6} \mathrm{H}_{14}$-AcOEt em gradiente crescente de polaridade resultou na obtenção do ácido vanílico (5, $25 \mathrm{mg})$.

Simiarenol (1): RMN ${ }^{1} \mathrm{H}\left(200 \mathrm{MHz}, \mathrm{CDCl}_{3}\right)$ : $\delta$ 3,46 (sl, H-3), 5,16 (ddd, $J=6,0 ; 2,0 ; 2,0, \mathrm{H}-6$ ), 1,04 (s, H-23), 1,14 (s, H-24), 0,89 (s, H-25), 1,00 (s, H-26), 0,92 (s, H-27), 0,78 (s, H-28), 0,88 (d, $J=6,7, \mathrm{H}-29$ ), $0,82$ (d, $J=6,7, \mathrm{H}-30)$. RMN ${ }^{13} \mathrm{C}\left(50 \mathrm{MHz}, \mathrm{CDCl}_{3}\right)$ : $\delta$ 18,03 (C-1), 27,73 (C-2), 76,37 (C-3), 40,79 (C-4), 141,93 (C-5), 121,98 (C-6), 24,01 (C-7), 44,20 (C-8), 34,79 (C-9), 50,19 (C-10), 34,10 (C-11), 28,95 (C-12), 38,56 (C-13), 39,26 (C-14), 29,06 (C-15), 35,36 (C-16), 42,75 (C-17), 51,70 (C-18), 19,85 (C-19), 28,28 (C-20), 59,99 (C-21), 30,75 (C-22), 29,04 (C-23), 25,44 (C-24), 17,84 (C-25), 15,71 (C-26), 14,97 (C-27), 16,04 (C-28), 21,93 (C-29) e 22,89 (C-30).

\section{RESULTADOS E DISCUSSÃO}

Fracionamentos cromatográficos da fração neutra em hexano das folhas conduziu ao isolamento de um triterpeno pentacíclico (1), um diterpeno (2) e de dois fitoesteróides (3-4), enquanto que da fração em diclorometano do caule foi isolado um benzenóide (5).

$\mathrm{O}$ composto 1 foi identificado como sendo um triterpeno pentacíclico com base na análise dos

dados obtidos dos espectros IV, EM e RMN, incluindo DEPT e HMQC, bem como pela comparação com dados da literatura. O espectro de absorção na região IV, obtido em KBr, revelou bandas de absorção indicativas da presença de grupo hidroxila (3507 e $1171 \mathrm{~cm}^{-1}$ ), de ligação dupla $\left(1639 \mathrm{~cm}^{-1}\right)$, além de bandas de para grupos alquílicos saturados (2927, 2842, 1450, $\left.1381 \mathrm{~cm}^{-1}\right)$. Os dados do espectro de $\mathrm{RMN}{ }^{1} \mathrm{H}$, registrado a $200 \mathrm{MHz}$ em $\mathrm{CDCl}_{3}$, permitiram identificar sinais, cujos valores de deslocamentos químicos estão condizentes com a presença de uma ligação dupla [ $\delta 5,59(\mathrm{~m}, \mathrm{H}-6,1 \mathrm{H})]$, um hidrogênio oximetínico [ $\delta 3,45$ (sl, H-3, $1 \mathrm{H})]$, um grupo isopropila $[\delta 0,80(d, J=6,6 \mathrm{~Hz})$ e $\delta 0,86(d, J=$ $6,4 \mathrm{~Hz})]$ e para seis grupos metílicos angulares $[\delta 0,75$; 0,87; 0,90; 0,98; 1,02 e 1,11 (s, cada)]. Os dados obtidos do espectro de $\mathrm{RMN}^{13} \mathrm{C}$-APT a $50 \mathrm{MHz}$ permitiram identificar sinais para um total de vinte e nove átomos de carbono (seis não hidrogenados, seis monoidrogenados, nove diidrogenados e oito triidrogenados). Dentre esses carbonos foram reconhecidos sinais cuja natureza e valores de deslocamentos químicos estão coerentes com a presença de uma ligação dupla do tipo trissubstituída [ $\delta 141,94(\mathrm{C}-5, \mathrm{C})$ e $\delta 121,98$ (C-6, CH)], de um grupo isopropila [ $\delta 21,93\left(\mathrm{C}-29, \mathrm{CH}_{3}\right), \delta 22,89\left(\mathrm{C}-30, \mathrm{CH}_{3}\right) \mathrm{e}$ $\delta 30,75$ (C-22, CH)] e para seis metilas $(\delta 29,04 ; 25,44$; $17,84 ; 15,71 ; 14,97$ e 16,04$)$. O espectro de RMN ${ }^{13} \mathrm{C}$-APT não evidenciou a presença do sinal referente ao carbono oximetínico sugerido pelos espectros de $\mathrm{RMN}^{1} \mathrm{H}$ e IV. Entretanto, no espectro bidimensional de correlação heteronuclear HMQC foram observadas correlações entre o sinal do hidrogênio que absorve em $\delta 5,59$ (H-6) e com o sinal em $\delta 121,98$ (C-6) e entre o sinal em $\delta$ $3,45(\mathrm{H}-3)$ e o sinal do carbono que absorve em $\delta 76,37$ (C-3), cujo valor de deslocamento químico coincide com o sinal residual do solvente utilizado. Esta informação possibilitou a inclusão na molécula de $\mathbf{1}$ de um carbono oximetínico. O espectro de massas obtido no modo positivo por ionização química à pressão atmosférica (APCI), mostrou o pico correspondente ao íon molecular em $\mathrm{m} / \mathrm{z} 427(\mathrm{M}+1)$ e um pico em $\mathrm{m} / \mathrm{z} 409\left(\mathrm{M}+1-\mathrm{H}_{2} \mathrm{O}\right)$ proveniente da perda, a partir do pico do íon molecular, de uma molécula de $\mathrm{H}_{2} \mathrm{O}$. Esta informação, associada à análise dos dados dos espectros de IV e RMN permitiu sugerir uma fórmula molecular de $\mathrm{C}_{30} \mathrm{H}_{50} \mathrm{O}(\mathrm{IDH}=6)$, compatível com o esqueleto de um triterpeno pentacíclico contendo uma ligação dupla, um grupo hidroxila e um grupo isopropila. A análise comparativa dos dados espectrais obtidos, especialmente dos deslocamentos químicos atribuídos aos grupos metilas e isopropila, ligação dupla e de C-17, C-18, C-19 e C-21, possibilitou excluir os esqueletos hopano, arborano, fernano, hancokinol, filicano e neogammacerano e sugerir para $\mathbf{1}$ um triterpeno pentacíclico da série adianano, conhecido como simiarenol. Embora esta substância ocorra com freqüência em algumas famílias de plantas, esta sendo relatado pela primeira vez na família Polygonaceae.

As estruturas dos compostos trans-fitol 
(2) (Sims \& Pettus Jr., 1976; Goodman et al., 1973), sitostenona (3), sitosterol (4) (Della Greca et al., 1990) e ácido vanílico (5) (Lai et al., 1985) foram identificadas através da comparação dos dados espectrais obtidos com os da literatura. Em Polygonaceae, a ocorrência deste ácido está restrita aos gêneros Polygonum (Adinarayanda et al., 1980; Ulubelen et al., 1992; Smorlarz, 2000) e Rumex (Djurdjevic et al., 1992). Sendo, portanto o primeiro relato no gênero Coccoloba.

\section{AGRADECIMENTOS}

Os autores agradecem ao CNPq, FAPEAL, MCT-IMSEAR e BNB-RENORBIO pelo apoio financeiro e pela Bolsa de Doutorado (P.E.S.O.), ao técnico Vicente Carlos C. de Oliveira (Laboratório de Tecnologia Farmacêutica da Universidade Federal da Paraíba), aos Profs. Drs. Edilberto R. Silveira (CENAUREMN da Universidade Federal do Ceará) e Jorge M. David (Instituto de Química da Universidade Federal da Bahia), pelos espectros de RMN e APCI.

\section{REFERÊNCIAS}

Adinarayanda D, Ramachandraiah P, Syamasundar KV 1980. Polyphenolic constituents of Polygonum glabrum seeds admixed with flowers and stem. Leather Sci 27: 268-270.

Agra MF, França PF, Barbosa-Filho JM 2007. Synopsis of the plants known as medicinal and poisonous in Northeast of Brazil. Rev Bras Farmacogn 17: 114140.

Agra MF, Silva KN, Basílio IJLD, França PF, Barbosa-Filho JM 2008. Survey of medicinal plants used in the region Northeast of Brazil. Rev Bras Farmacogn 18: 472-508.

Antoun MD, Gerena L, Milhous WK 1993. Screening of the flora of Puerto Rico for potential antimalarial bioactives. Int J Pharmacogn 31: 255-258.

Barbosa-Filho JM, Medeiros KCP, Diniz MFFM, Batista LM, Athayde-Filho PF, Silva MS, Cunha EVL, Almeida JRGS, Quintans-Júnior LJ 2006a. Natural products inhibitors of the enzyme acetylcholinesterase. Rev Bras Farmacogn 16: 258-285.

Barbosa-Filho JM, Martins VKM, Rabelo LA, Moura MD, Silva MS, Cunha EVL, Souza MFV, Almeida RN, Medeiros IA 2006b. Natural products inhibitors of the angiotensin converting enzyme (ACE). A review between 1980-2000. Rev Bras Farmacogn 16: 421446.

Barbosa-Filho JM, Alencar AA, Nunes XP, Tomaz ACA, Sena-Filho JG, Athayde-Filho PF, Silva MS, Souza MFV, da-Cunha EVL 2008. Sources of alpha-, beta-, gamma-, delta- and epsilon-carotenes: A twentieth century review. Rev Bras Farmacogn 18: 135-154.

Barros IB, Fidelis QC, Santos GF, Pinto JP, Faccione M, Tsuboy MSF, Montovani MS, Braz-Filho R, Ferreira DT 2007. Phytochemistry of root and leaf extracts of Coccoloba mollis. $1^{\text {st }}$ Brazilian Conference on Natural Products and XXVII Annual Meeting on
Micromolecular Evolution, Systematics and Ecology. São Pedro/SP, Brasil.

Barroso GM 1978. Sistematica de Angiospermas do Brasil. Rio de Janeiro: Livros Técnicos e Científicos.

Cardoso CAL, Honda NK, Dias ES 2006. Avaliação do perfil cromatográfico em espécies de Polygonum e amostras comercializadas como "erva-de-bicho". Rev Bras Farmacogn 16: 236-245.

Coe FG, Anderson GJ 1996. Screening of medicinal plants used by the garifuna of Eastern Nicaragua for bioactive compounds. J Ethnopharmacol 53: 29-50.

Compagnone R, Castillo Suarez A, Delle Monache F 1995. Myricetin-3-O-rhamnoside from the leaves and twigs of Coccoloba dugandiana. Rev Colomb Quim 24: 65-68.

Cota BB, Oliveira AB, Souza-Filho JD, Braga FC 2003. Antimicrobial activity and constituents of Coccoloba acrostichoides. Fitoterapia 74: 729-731.

Dan S, Dan SS 1986. Phytochemical study of Adansonia digitata, Coccoloba excoriata, Psychotria adenophylla and Schleichera oleosa. Fitoterapia 57: 445-446.

Della Greca M, Monaco P, Previtera L 1990. Stigmasterols from Typha latifolia. J Nat Prod 53: 1430-1435.

Djurdjevic L, Culafic L, Kozomara B, Kof E, Kefeli V 1992. Phenol content in relation to sex expression in sorreal. Fiziologiya i Biokhimiya Kul'turnykh Rastenii 24: 64-68; CA 116:232039.

Falcão HS, Lima IO, Santos VL, Dantas HF, Diniz MFFM, Barbosa-Filho JM, Batista LM 2005. Review of the plants with anti-inflammatory activity studied in Brazil. Rev Bras Farmacogn 15: 381-391.

Ferreira DT, Pinto JP, Fidelis QC, Santos GF, Braz-Filho R 2006. Antraquinonas farmacologicamente ativas isoladas de Coccoloba mollis Casar - Polygonaceae. $30^{a}$ Reunião Anual da Sociedade Brasileira de Química, Águas de Lindóia/SP, Brasil.

Frisbey A, Roberts JM, Ennings JC, Gottshall RY, Lucas EH 1953. The occurrence of antibacterial substances in seed plants with special reference to Mycobacterium tuberculosis (Third report). Mich State Univ Agr Appl Sci Quart Bull 35: 392-404.

Goodman RA, Oldfield E, Allerhand A 1973. Assignments in the natural-abundance carbon-13 nuclear magnetic resonance spectrum of chlorophyll A and a study of segmental motion in neat phytol. J Amer Chem Soc 95: 7553-7558.

Halberstein RA, Saunders AB 1978. Traditional medical practices and medicinal plant usage on a Bahamian Island. Cult Med Psychiatry 2: 177-203.

Jácome RLRP, Lopes DES, Recio RA, Macedo JF, Oliveira AB 2004. Caracterização farmacognóstica de Polygonum hodropiperoides Michaux e P. spectabile Mart. (Polygonaceae). Rev Bras Farmacogn 14: 2127.

Keiser I, Harris EJ, Miyashita DH, Jacobson M, Perdue RE 1975. Attraction of ethyl ether extracts of 232 botanicals to oriental fruit flies, melon flies, and Mediterranean fruit flies. Lloydia 38: 141-152.

Lai A, Monduzzi M, Saba G 1985. Carbon-13 NMR studies on catechol, phenol and benzene derivatives of biological relevance. Magn Reson Chem 23: 379383. 
Li XC, Elsohly HN, Nimrod AC, Clark AM 1999. Antifungal activity of (-)-epigallacatechin gallate from Coccoloba dugandiana. Planta Med 65: 780.

Lorenzi H. 1998. Árvores Brasileiras: Manual de Identificação e Cultivo de Plantas Arbóreas do Brasil. Nova Odessa: Instituto Plantarum.

Melo E 1999. Levantamenyo da família Polygonaceae no estado da Bahia: espécies do semi-árido. Rodriguésia 50: 29-47.

Melo E 2000. Polygonaceae da Cadeia do Espinhaço, Brasil. Acta Bot Bras 14: 273-300.

Melo E 2004. As espécies de Coccoloba P. Browne (Polygonaceae) da Amazônia Brasileira. Acta Amazônica 34: 525-551.

Morton JF 1977. Medicinal and other plants used by people on North Caicos (Turks and Caicos Islands, West Indies). Int J Crude Drug Res 15: 1-24.

Perez S, Zavala MA, Arias L, Perez C, Perez RM 2001. Antimicrobial study of bark from five tree species. Phytother Res 15: 356-359.

Shaw PE, Moshonas MG, Baldwin EA 1992. Volatile constituents of Coccoloba uvifera. Phytochemistry 31: 3495-3497.

Sims JJ, Pettus Jr JA 1976. Isolation of free cis and transphytol from the red alga Gracilaria andersoniana. Phytochemistry 15: 1076-1077.

Smolarz HD 2000. Chromatographical analysis of phenolic acids in some species of Polygonum L. genus. Part 2. Quantitative determination of the major components by high performance liquid chromatography (HPLC). Acta Soc Bot Poloniae 69: 21-23.

Sousa MP, Rouquayrol MZ 1974. Molluscicidal activity of plants from Northeast Brazil. Rev Bras Pesq Med Biol 7: 389-394.

Ulubelen A, Tan N, Ucer M 1992. Flavonoids from Polygonum cognatum. Fitoterapia 63: 87.

Wall ME, Wani MC, Hughes TJ, Taylor H 1988. Plant antimutagenic agents, 1 . General bioassay and isolation procedures. J Nat Prod 51: 866-873.

Wall ME, Wani MC, Brown DM, Fullas F, Oswald JB, Josephson FF, Thornton NM, Pezzuto JM, Beecher CWW, Farnsworth NR, Cordell GA, Kinghorn AD 1996. Effect of tannins on screening of plant extracts for enzyme inhibitory activity and techniques for their removal. Phytomedicine 3: 281-285.

Xing-Cong L, El Sohly H, Nimrod AC, Clark AM 1999. Antifungal activity of (-)-epigallocatechin gallate from Coccoloba dugandiana. Planta Med 65: 780.

Zamora-Martinez MC, Pola CNP 1992. Medicinal plants used in some rural populations of Oaxaca, Puebla and Veracruz, Mexico. J Ethnopharmacol 35: 229-257. 